\title{
BOUNDS FOR RATIOS OF EIGENVALUES OF THE DIRICHLET LAPLACIAN
}

\author{
MARK S. ASHBAUGH AND RAFAEL D. BENGURIA
}

(Communicated by Barbara L. Keyfitz)

\begin{abstract}
We use a doubling scheme to derive a bound for the ratio of the $2^{k}$ th eigenvalue to the first for the Dirichlet Laplacian on a bounded domain $\Omega \subset \mathbb{R}^{n}$. The explicit bounds we obtain derive from the optimal bound $\left(\lambda_{2} / \lambda_{1}\right)_{\Omega} \leq\left(\lambda_{2} / \lambda_{1}\right)_{n \text {-dimensional ball (the Payne-Pólya-Weinberger conjecture) re- }}$ cently proved by us.
\end{abstract}

Consider the Dirichlet problem on a bounded domain $\Omega \subset \mathbb{R}^{n}$ :

$$
\begin{array}{rlrl}
-\Delta u & =\lambda u & & \text { in } \Omega, \\
u=0 & & \text { on } \partial \Omega .
\end{array}
$$

Let its eigenvalues (known to be positive) be denoted by

$$
\lambda_{1}<\lambda_{2} \leq \lambda_{3} \leq \cdots
$$

with a corresponding orthonormal set of eigenfunctions denoted by $u_{i}$ for $i=$ $1,2,3, \ldots$. We prove

Theorem. Let $k$ be an integer larger than 1 . Then

$$
\frac{\lambda_{2^{k}}}{\lambda_{1}}<\left(\left.\frac{\lambda_{2}}{\lambda_{1}}\right|_{n \text {-dimensional ball }}\right)^{k}=\left(\frac{j_{n / 2,1}^{2}}{j_{n / 2-1,1}^{2}}\right)^{k} .
$$

Remarks. We follow Abramowitz and Stegun [1] in denoting the $k$ th positive zero of the Bessel function $J_{p}(x)$ by $j_{p, k}$. Our result is much better than the bound

$$
\frac{\lambda_{m}}{\lambda_{1}} \leq\left(1+\frac{4}{n}\right)^{m-1}
$$

which follows from the bound

$$
\frac{\lambda_{m+1}}{\lambda_{m}} \leq 1+\frac{4}{n}
$$

of Payne-Pólya-Weinberger [15, 16] and Thompson [18] for ratios of consecutive eigenvalues. First, our constant $j_{n / 2,1}^{2} / j_{n / 2-1,1}^{2}$ is less than $1+4 / n$ (in two

Received by the editors August 13, 1992.

1991 Mathematics Subject Classification. Primary 35P15; Secondary 35J05.

The first author was supported in part by NSF grants DMS-9114162 and INT-9123481.

The second author was supported in part by FONDECYT (Chile) project number 1238-90. 
dimensions, for example, we have approximately 2.539 as opposed to 3 ) and, second and more importantly, our bound for $\lambda_{m} / \lambda_{1}$ grows as a constant to the power $\log _{2} m$ rather than as a constant to something linear in $m$. (We should mention, to be fair, that the approach we present below could be used in conjunction with the PPW bound $\lambda_{2} / \lambda_{1} \leq 1+4 / n$ to obtain $\lambda_{2^{k}} / \lambda_{1} \leq(1+4 / n)^{k}$.) On the other hand, for large $m$ our bound for $\lambda_{m} / \lambda_{1}$ still falls well short of the behavior $\mathrm{cm}^{2 / n}$, the known asymptotic form for $\lambda_{m} / \lambda_{1}$ (Weyl $[19,20]$ ). This is not surprising given that our bound is based only on the (best) bound for $\lambda_{2} / \lambda_{1}$ : one can construct examples where $\lambda_{m+1} / \lambda_{m}$ is as near as one wants to the value $j_{n / 2,1}^{2} / j_{n / 2-1,1}^{2}$ but such large ratios of consecutive eigenvalues cannot be maintained in the long term. To put our result in its best light one should view it not as an asymptotic result but as a rather stringent enforcement of the fact that no domain can have strings of consecutive eigenvalues such that each individual ratio $\lambda_{m+1} / \lambda_{m}$ is close to what it could be if that ratio alone were being maximized. Our result, though, is almost certainly not optimal even in this sense (e.g., the best bound for $\lambda_{4} / \lambda_{1}$ is probably somewhat less than our value $\left(j_{n / 2,1}^{2} / j_{n / 2-1,1}^{2}\right)^{2}$; cf. our discussion of this point in [7]).

Proof of the Theorem. First we introduce some notation. Since in our proof we shall use many subdomains of $\Omega$, we adopt the notation $\lambda_{i}(D), u_{i}(D)$ to denote the $i$ th eigenvalue and corresponding eigenfunction of $D$, where as above the $u_{i}$ 's are taken as an orthonormal set. Only for $\Omega$ itself do we leave off this indication of domain-dependence.

Our doubling scheme goes as follows. Start with $\Omega$ and use the two nodal domains of $u_{2}$ as new subdomains $\Omega_{1}^{(1)}, \Omega_{2}^{(1)}$. (That $u_{2}$ has two nodal domains is a direct consequence of Courant's nodal domain theorem, see [12, 10] for details.) Then in $\Omega_{1}^{(1)}$ and $\Omega_{2}^{(1)}$ we use the nodal domains of $u_{2}\left(\Omega_{1}^{(1)}\right)$ and $u_{2}\left(\Omega_{2}^{(1)}\right)$ to arrive at four new subdomains $\Omega_{1}^{(2)}, \Omega_{2}^{(2)}, \Omega_{3}^{(2)}, \Omega_{4}^{(2)}$. In general, after $k$ such subdivisions one arrives at $2^{k}$ subdomains

$$
\Omega_{1}^{(k)}, \Omega_{2}^{(k)}, \ldots, \Omega_{2^{k}}^{(k)},
$$

where we agree to arrange them so that

$$
\overline{\Omega_{j}^{(k-1)}}=\overline{\Omega_{2 j-1}^{(k)}} \cup \overline{\Omega_{2 j}^{(k)}} .
$$

Since our domains $\Omega_{j}^{(k)}$ are disjoint for $j=1,2, \ldots, 2^{k}$ and $\bar{\Omega}$ is the union of their closures we have

$$
H_{0}^{1}\left(\Omega_{1}^{(k)}\right) \oplus \cdots \oplus H_{0}^{1}\left(\Omega_{2^{k}}^{(k)}\right) \subset H_{0}^{1}(\Omega),
$$

where $H_{0}^{1}(D)$ is used to denote the indicated Sobolev space of functions on the domain $D$. For each $j=1, \ldots, 2^{k}$ we can use the function $u_{1}\left(\Omega_{j}^{(k)}\right)$ as a trial function in $H_{0}^{1}(\Omega)$ if we agree to consider it as a function on $\Omega$ which is supported in $\overline{\Omega_{j}^{(k)}}$ and is 0 elsewhere. Then from the $2^{k}$ linearly independent functions $u_{1}\left(\Omega_{j}^{(k)}\right) \in H_{0}^{1}(\Omega)$ for $j=1, \ldots, 2^{k}$ we can construct a nontrivial linear combination

$$
\varphi=\sum_{j=1}^{2^{k}} c_{j} u_{1}\left(\Omega_{j}^{(k)}\right)
$$


which is orthogonal to the first $2^{k}-1$ eigenfunctions of $-\Delta$ on $\Omega$, i.e., to $u_{1}, \ldots, u_{2^{k}-1}$. This makes $\varphi$ an appropriate trial function for bounding $\lambda_{2^{k}}$ via the Rayleigh-Ritz inequality

$$
\lambda_{2^{k}} \leq \frac{\int|\nabla \varphi|^{2}}{\int \varphi^{2}}
$$

Now by the fact that $\Omega_{i}^{(k)} \cap \Omega_{j}^{(k)}=\varnothing$ for $i \neq j$ it is clear that the $u_{1}\left(\Omega_{j}^{(k)}\right)$ 's form an orthonormal set in $H_{0}^{1}(\Omega)$ for $j=1, \ldots, 2^{k}$ and, moreover, that

$$
\int \varphi^{2}=\sum_{j=1}^{2^{k}} c_{j}^{2}
$$

and

$$
\int|\nabla \varphi|^{2}=\sum_{j=1}^{2^{k}} \lambda_{1}\left(\Omega_{j}^{(k)}\right) c_{j}^{2} .
$$

It is then immediate that

$$
\lambda_{2^{k}} \leq \max _{1 \leq j \leq 2^{k}} \lambda_{1}\left(\Omega_{j}^{(k)}\right)
$$

and then, by the way we generated the $\Omega_{j}^{(k)}$,s, that

$$
\lambda_{2^{k}} \leq\left(\sup _{\Omega^{\prime} \subset \mathbb{R}^{n}} \frac{\lambda_{2}}{\lambda_{1}}\right) \max _{1 \leq j \leq 2^{k-1}} \lambda_{1}\left(\Omega_{j}^{(k-1)}\right) .
$$

This follows because we have

$$
\lambda_{2}\left(\Omega_{j}^{(k-1)}\right)=\lambda_{1}\left(\Omega_{2 j-1}^{(k)}\right)=\lambda_{1}\left(\Omega_{2 j}^{(k)}\right) .
$$

Continuing in this fashion we arrive at

$$
\frac{\lambda_{2^{k}}}{\lambda_{1}} \leq\left(\sup _{\Omega^{\prime} \subset \mathbb{R}^{n}} \frac{\lambda_{2}}{\lambda_{1}}\right)^{k}
$$

which is inequality (3) of our Theorem, by virtue of our proof $[4,5,8]$ of the Payne-Pólya-Weinberger conjecture.

Remarks. (1) One can stop one iteration short of the end in our proof above to obtain

$$
\frac{\lambda_{2^{k}}}{\lambda_{2}} \leq\left(\frac{j_{n / 2,1}^{2}}{j_{n / 2-1,1}^{2}}\right)^{k-1}
$$

and, more generally, if one first divides according to the nodal domains of $u_{m}$ and then uses our doubling scheme $k$ times one arrives at

$$
\frac{\lambda_{2^{k} l(m)}}{\lambda_{m}} \leq\left(\frac{j_{n / 2,1}^{2}}{j_{n / 2-1,1}^{2}}\right)^{k},
$$

where $l(m)$ is the function giving the number of nodal domains of $u_{m}$. This more general inequality is not universal since it contains the domain-dependent function $l(m)$; indeed, for $m \geq 3$ it can (possibly) improve upon (16) only 
if $l(m)>2$, which is known to not hold in general (for example, for the $n$ dimensional ball one has $l(3)=\cdots=l(n+1)=2$ or see the example given by Courant-Hilbert [12, pp. 455-456]). Note that the $m=1$ case of (17) is inequality (3), while the $m=2$ case is (16) (with $k+1$ replacing $k$ ).

(2) One can improve our bounds (3), (16), and (17) to strict inequalities in the following cases:
inequality (3) is strict for $k \geq 2$,
inequality (16) is strict for $k \geq 2$,
inequality (17) is strict for $m \geq 2$ and $k \geq 1$.

These follow from the fact that if $\lambda_{2} / \lambda_{1}=j_{n / 2,1}^{2} / j_{n / 2-1,1}^{2}$ for some domain in $n$ dimensions then that domain is an $n$-dimensional ball. Hence if the doubling scheme is applied two or more times we can be sure that at least one of them produces a strict inequality. In the latter two cases for the lowest values of $k$ allowed above one needs a slightly more involved argument (see [7] for one such argument).

(3) As in [7], one could base an alternative proof of the Theorem on the monotonicity property of eigenvalues with respect to added Dirichlet conditions, in particular, restricting our functions to be 0 on the boundaries of all the $\Omega_{j}^{(k)}$,s for $j=1, \ldots, 2^{k}$. This approach yields immediately

$$
\lambda_{2^{k}} \leq \max _{1 \leq j \leq 2^{k}} \lambda_{1}\left(\Omega_{j}^{(k)}\right)
$$

i.e., inequality (12), but from this point of view one cannot obtain strict inequalities as done in Remark (2) above without getting into the more detailed considerations mentioned there.

(4) One could obtain results analogous to those above by taking nodal domains of $u_{m}(D)$, where $D$ is a subdomain and iterating the process (much as was done above using $u_{2}(D)$ 's). However, because $l(m)$ can be less than $m$ (and is, except for finitely many choices of $m$; see Pleijel [17] or, for example, Bandle [10] or Kreith [13]) this procedure does not lead to a tripling, quadrupling, ..., scheme. Moreover, since $\sup _{\Omega \subset \mathbb{R}^{n}} \lambda_{m} / \lambda_{1}$ is not explicitly known for $m \geq 3$, to get numerical values one would typically have to replace this quantity, which will appear to the $k$ th power if $k$ iterations of the process are used, by an upper bound for it. Aside from the case of $\lambda_{3} / \lambda_{1}$ (see Marcellini [14] for the best constant to date for two dimensions and [9] for a discussion in higher dimensions) little is known in this direction except for what one gets from our approach above and in [7]. For these two reasons (the first of which is by far the more compelling) we leave aside the presentation of these other results.

(5) Our ratio results above can also be read as giving lower bounds to the counting function $N(\lambda)$, where $N(\lambda)$ denotes the number of eigenvalues less than or equal to $\lambda$. For $\lambda \geq \lambda_{1}$ one has from inequality (3)

$$
N(\lambda) \geq 2^{\left\lfloor\log \left(\lambda / \lambda_{1}\right) / \log \left(j_{n / 2,1}^{2} / j_{n / 2-1,1}^{2}\right)\right\rfloor}
$$

for $n \geq 2$, and, similarly, from (16) one obtains

$$
N(\lambda) \geq 2^{\left\lfloor\log \left(\lambda / \lambda_{2}\right) / \log \left(j_{n / 2,1}^{2} / j_{n / 2-1,1}^{2}\right)\right\rfloor+1} .
$$

Here $\lfloor\cdot\rfloor$ denotes the greatest integer function, i.e., $\lfloor x\rfloor$ denotes the largest integer less than or equal to $x$. These bounds also apply if $\lambda_{1}$ (respectively $\lambda_{2}$ ) on the righthand side is replaced by an upper bound. 
(6) All the results presented above can be carried over to the eigenvalues of the Schrödinger operator $H=-\Delta+V(x)$ acting on a bounded domain in $\mathbb{R}^{n}$ with Dirichlet boundary conditions and a nonnegative potential $V$. In addition, with suitable modification our results can be extended to more general elliptic eigenvalue problems. Since these extensions are straightforward, we only refer the reader to our papers $[5,6,7]$ where background material and other comments appear. For Schrödinger operators with nonnegative potentials the one-dimensional case is also of interest. Indeed, this is the setting in which we first studied eigenvalue ratios, and many of the ideas discussed above have precursors in this setting. For example, the arguments mentioned in Remark (3) are paralleled to some extent by an alternative approach for the one-dimensional case that we presented earlier [3] (see also our remarks in the last section of [7]) which was based on a result of Barnsley [11]. Also, in connection with Remark (4), it should be noted that in the one-dimensional setting doubling, tripling, $\ldots$, schemes all not only work but produce optimal bounds. This follows in part from the fact that one always has $l(m)=m$ in one dimension. In particular, the doubling and tripling schemes in one dimension were mentioned in our papers $[2,3]$ where it was noted that $\lambda_{2} / \lambda_{1} \leq 4$ and $\lambda_{3} / \lambda_{1} \leq 9$ lead to $\lambda_{m} / \lambda_{1} \leq m^{2}$ for all integers of the form $m=2^{a} 3^{b}$, where $a$ and $b$ are nonnegative integers. In [3] we also gave a proof of $\lambda_{m} / \lambda_{1} \leq m^{2}$ for $m=2,3, \ldots$ which was based on a Prüfer transformation and a differential comparison argument.

NOTE ADDED IN PROOF (10/4/93)

In connection with our reference (in Remark (4) above) to Marcellini's upper bound for $\lambda_{3} / \lambda_{1}$ being the best to date, we note that recently we have obtained a small improvement. Our improved bound and related results will appear in a forthcoming paper entitled The range of values of $\lambda_{2} / \lambda_{1}$ and $\lambda_{3} / \lambda_{1}$ for the fixed membrane problem.

\section{ACKNOWLEDGMENT}

The first author thanks the Physics and Mathematics Departments of the Pontificia Universidad Católica de Chile for hospitality while this work was completed. Financial support from the Universidad Católica, CONICYT, and NSF is also gratefully acknowledged.

\section{REFERENCES}

1. M. Abramowitz and I. A. Stegun, eds., Handbook of mathematical functions, National Bureau of Standards Applied Mathematics Series, vol. 55, U.S. Government Printing Office, Washington, D.C., 1964.

2. M. S. Ashbaugh and R. D. Benguria, Optimal lower bounds for eigenvalue gaps for Schrödinger operators with symmetric single-well potentials and related results, Maximum Principles and Eigenvalue Problems in Partial Differential Equations (Philip W. Schaefer, ed.), Pitman Res. Notes Math. Ser., vol. 175, Longman Sci. Tech., Harlow, 1988, pp. 134-145.

3. __ Optimal bounds for ratios of eigenvalues of one-dimensional Schrödinger operators with Dirichlet boundary conditions and positive potentials, Comm. Math. Phys. 124 (1989), 403-415.

4. _ Proof of the Payne-Polya-Weinberger conjecture, Bull Amer. Math. Soc. (N.S.) 25 (1991), 19-29. 
5. $ـ$ A sharp bound for the ratio of the first two eigenvalues of Dirichlet Laplacians and extensions, Ann. of Math. (2) 135 (1992), 601-628.

6. _ Isoperimetric bound for $\lambda_{3} / \lambda_{2}$ for the membrane problem, Duke Math. J. 63 (1991), 333-341.

7. $\ldots$ Isoperimetric bounds for higher eigenvalue ratios for the n-dimensional fixed membrane problem, Proc. Roy. Soc. Edinburgh (to appear).

8. $\ldots$ A second proof of the Payne-Polya-Weinberger conjecture, Comm. Math. Phys. 147 (1992), 181-190.

9. __ More bounds on eigenvalue ratios for Dirichlet Laplacians in $n$ dimensions, SIAM J. Math. Anal. (1993) (to appear).

10. C. Bandle, Isoperimetric inequalities and applications, Monographs Stud. Math., vol. 7, Pitman, Boston, 1980.

11. M. F. Barnsley, Lower bounds for quantum-mechanical energy levels, J. Phys. A 11 (1978), 55-68.

12. R. Courant and D. Hilbert, Methods of mathematical physics, Vol. I, Wiley Interscience, New York, 1953.

13. K. Kreith, The nodal number of a domain, Amer. Math. Monthly 78 (1971), 181-182.

14. P. Marcellini, Bounds for the third membrane eigenvalue, J. Differential Equations 37 (1980), 438-443.

15. L. E. Payne, G. Pólya, and H. F. Weinberger, Sur le quotient de deux fréquences propres consécutives, C. R. Acad. Sci. Paris 241 (1955), 917-919.

16. $\_$, On the ratio of consecutive eigenvalues, J. Math. and Phys. 35 (1956), 289-298.

17. A. Pleijel, Remarks on Courant's nodal line theorem, Comm. Pure Appl. Math. 9 (1956), 543-550.

18. C. J. Thompson, On the ratio of consecutive eigenvalues in $n$ dimensions, Stud. Appl. Math. 48 (1969), 281-283.

19. H. Weyl, Über die asymptotische Verteilung der Eigenwerte, Nachr. Königl. Ges. Wiss. Göttingen (1911), 110-117.

20. __ Das asymptotische Verteilungsgesetz der Eigenwerte linearer partieller differentialgleichungen (mit einer Anwendung auf die Theorie der Hohlraumstrahlung), Math. Ann. 71 (1912), 441-479.

Department of Mathematics, University of Missouri, Columbia, Missouri 65211

E-mail address: mathmsa@mizzou1.missouri.edu

Facultad de Fisica, Pontificia Universidad Católica de Chile, Casilla 306, Correo 22, Santiago, Chile

E-mail address: rbenguri@lascar.puc.cl 